\title{
Primary Lymphoma Isolated to the Trigeminal Nerve
}

\author{
Andrew S. Jack, Cameron M. McDougall, J. Max Findlay
}

Can J Neurol Sci. 2014; 41: 103-105

Primary central nervous system lymphoma (PCNSL) is a malignant lymphoma located solely in the nervous system. The majority of PCNSL arises from white matter in the brain, although cases have also been reported originating outside the cerebrum in Meckel's cave and the cavernous sinus ${ }^{1-5}$. Its presentation as a facial pain syndrome and cranial neuropathy is rare $^{3}$. Here, we describe a case that required trigeminal nerve biopsy for diagnosis.

\section{Case}

A 57-year-old previously healthy man presented with a five month history of progressive and severe left-sided facial pain. Examination revealed hyperalgesia in the distribution of the first division of the trigeminal nerve. Normal initial investigations included computed tomography (CT), magnetic resonance imaging (MRI) (Figure 1A and B), and cerebrospinal fluid analysis. Medical treatment for trigeminal neuralgia was started, however this proved to be unsuccessful. His pain worsened, and repeat MRI showed an enlarged, non-enhancing lesion at the root entry zone of the trigeminal nerve (Figure 1C).

A neoplastic etiology was suspected, and the patient was investigated for metastatic disease. Normal studies included a CT of the neck, chest, abdomen, and pelvis, multiple lumbar punctures, two positron emission tomography (PET) studies, a bone marrow biopsy, and lab and hematological work-up for inflammatory and paraneoplastic causes.

Neurosurgical referral was then made for tumor biopsy. Via a retrosigmoid approach the nerve was found to be grossly

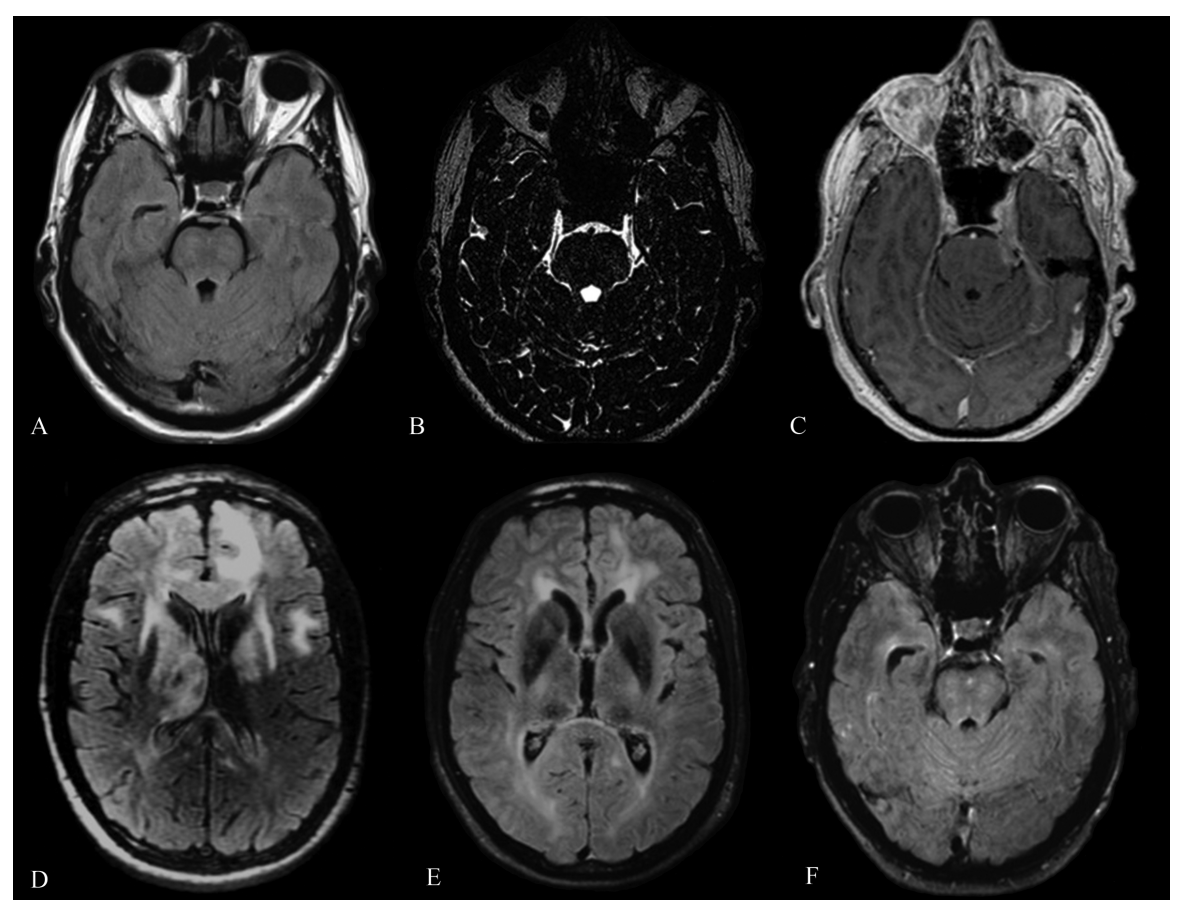

Figure 1: A) Axial slice of a fluid attenuated inversion recovery (FLAIR) sequence MRI with normal appearing left trigeminal nerve. B) Axial slice of a fat-saturated proton-density weighted $3 D$ transient-spin-echo (SPACE) sequence showing a normal appearing trigeminal nerve. C) Axial slice of a magnetization-prepared rapid acquisition with gradient echo (MP-RAGE) sequence showing an abnormal and homogenously enhancing left trigeminal nerve. D) Axial slice of a $3 D$ fast-spin-echo (CUBE) FLAIR sequence demonstrating T2 hyperintense signal change throughout the deep grey matter and frontal lobes bilaterally. E) Postchemotherapy FLAIR sequence showing dramatic improvement of changes seen in D).F) Post-chemotherapy MRI with resolution of changes seen in figure $C$ ).

\footnotetext{
From the Division of Neurosurgery, Department of Surgery, University of Alberta Hospital, Edmonton, Alberta, Canada.

Received April 22, 2013. Final Revisions Submitted July 10, 2013.

Correspondence to: Andrew S. Jack, Division of Neurosurgery, 2D1 WMC, University of Alberta, 8440 112 St, Edmonton, Alberta, T6G 2B7, Canada. Email: asjack@ualberta.ca.
} 


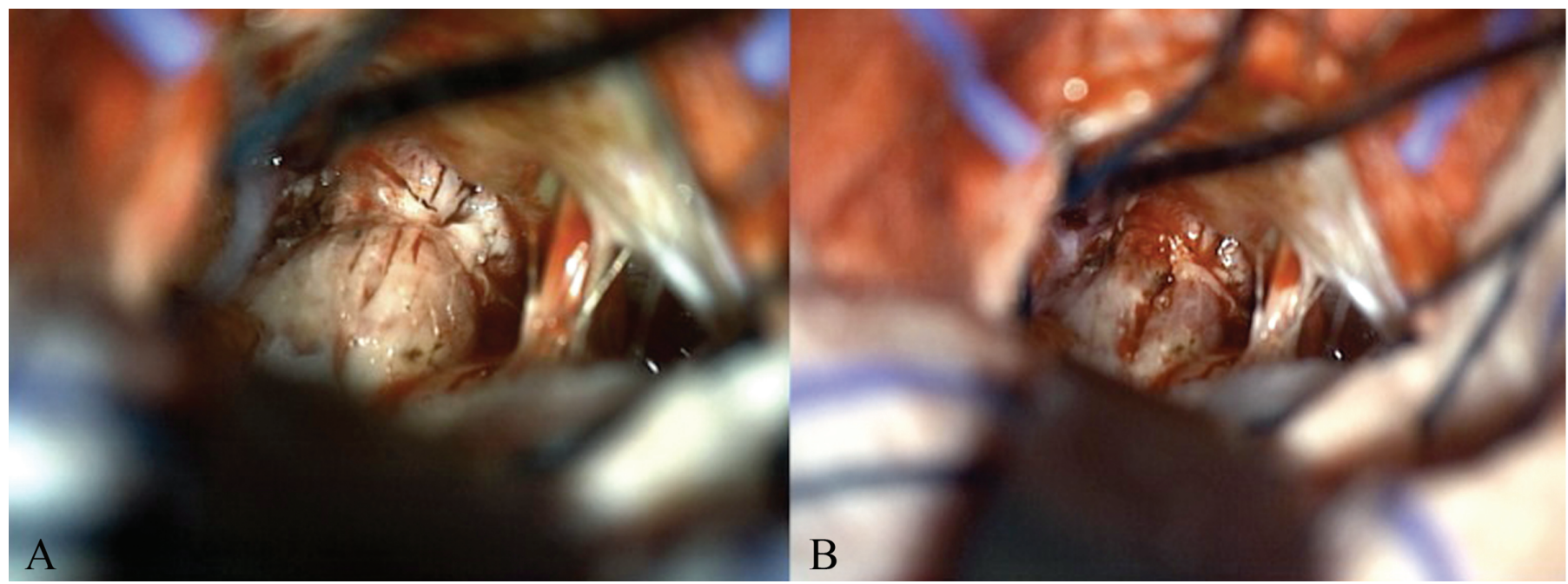

Figure 2: A) Operative photograph of a retrosigmoid approach with cerebellar retraction inferiorly showing an abnormal left trigeminal nerve. B) Post-biopsy image.

abnormal and enlarged with some mass effect on the adjacent pons (Figure 2A). The lesion was greyish-tan in color, and dense with no way to separate tumor and nerve. Biopsies were taken, and the frozen section specimens were consistent with malignancy. Post-operatively, the patient's condition deteriorated with the development of confusion, somnolence, and incontinence. The MRI revealed extensive, non-enhancing, signal change throughout the deep grey matter and frontal lobes bilaterally (Figure 1D). The final biopsy pathology showed a diffuse growth pattern with prominent infiltration of the nerve by atypical lymphocytes with centroblastic features (Figure 3A). Immunohistochemical studies showed staining of large cells by CD20 (Figure 3B), CD45, BCL2, and MUM-1 (B-cell markers). Also, $80-90 \%$ of the cells stained positive for Ki-67 with marker MIB-1 (Figure 3C) showing marked cellularity, in keeping with the diagnosis of diffuse large B-cell lymphoma (DLBCL).

After obtaining the diagnosis, the patient was started on chemotherapy including one cycle of methotrexate $\left(3.5 \mathrm{~g} / \mathrm{m}^{2}\right)$, procarbazine $\left(100 \mathrm{mg} / \mathrm{m}^{2}\right)$, and vincristine $\left(1.4 \mathrm{mg} / \mathrm{m}^{2}\right)$, followed by a second cycle of methotrexate $\left(3.5 \mathrm{~g} / \mathrm{m}^{2}\right)$, rituximab $\left(350 \mathrm{mg} / \mathrm{m}^{2}\right)$, and cytarabine $\left(2 \mathrm{~g} / \mathrm{m}^{2}\right)$. The patient responded well to this regimen with resolution of his pain and confusion. The MRI and PET studies showed resolution of the lymphoma (Figure 1E and F), and he went on to have an autologous stem cell transplant. At six month follow-up, he had made a good recovery from the transplant with examination finding only leftsided numbness in the V1 and V2 trigeminal distribution, and a weak corneal reflex.

\section{DisCUSSION}

Primary central nervous system lymphoma is usually of NonHodgkin B-cell origin, and typically arises in the periventricular white matter or deep nuclei. Few reports characterize skull base

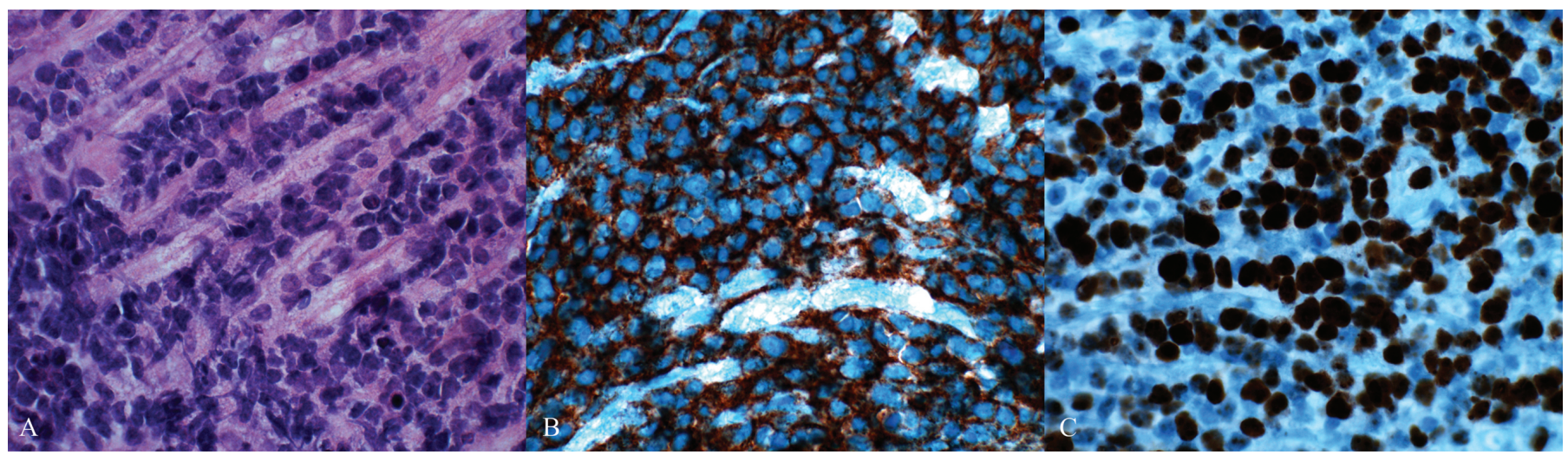

Figure 3: A) Hematoxylin and eosin stained pathological section (400x magnification) from the biopsy showing prominent infiltration of the nerve by atypical lymphocytes; B) immunohistochemical stained pathological section (400x magnification) demonstrating strong positive staining with CD20 in keeping with diffuse large B-cell lymphoma, and C), immunohistochemical stained pathological section (200x magnification) showing strong MIB-1 staining indicating marked cellular proliferation. 
lymphomatous tumors ${ }^{1-5}$, and even fewer describe primary lymphomatous tumors of the trigeminal nerve $e^{1,3}$. This case highlights a rare presentation of PCNSL as a facial pain syndrome with initial investigations being negative, and subsequent progression of the disease showing trigeminal nerve enhancement. This patient has done well with chemotherapy and stem-cell transplant, although relapse for these tumors is not uncommon. Close follow-up with the patient will be essential.

There are few reports describing the diagnostic difficulty of these lesions, with misdiagnoses of lymphomatous tumors as both schwannomas and meningiomas noted ${ }^{1,5}$. These cases have resulted in unnecessary, more extensive surgeries. Here, we selected a retrosigmoid approach for nerve biopsy, although percutaneous needle biopsy of Meckel's cave lesions has been previously described ${ }^{3}$. For tumors such as this one infiltrating into Meckel's cave, the percutaneous approach is a less invasive, and perhaps better alternative.

\section{Conclusion}

This case describes the rare occurrence of a primary trigeminal nerve lymphoma presenting as trigeminal neuralgia. Although this tumor was diagnosed via open biopsy of the nerve, it is important to first rule out other more common possibilities. These lesions present a significant diagnostic challenge, and it is important to keep lymphoma in the differential diagnosis as the role of surgery is limited to tissue diagnosis.

\section{REFERENCES}

1. Abdel Aziz KM, van Loveren HR. Primary lymphoma of Meckel's cave mimicking trigeminal schwannoma: case report. Neurosurgery. 1999;44:859-62; discussion 62-3.

2. Delpassand ES, Kirkpatrick JB. Cavernous sinus syndrome as the presentation of malignant lymphoma: case report and review of the literature. Neurosurgery. 1988;23:501-4.

3. Kinoshita $\mathbf{M}$, Izumoto $\mathrm{S}$, Oshino $\mathrm{S}$, et al. Primary malignant lymphoma of the trigeminal region treated with rapid infusion of high-dose MTX and radiation: case report and review of the literature. Surg Neurol. 2003;60:343-8; discussion 8.

4. Nakatomi H, Sasaki T, Kawamoto S, et al. Primary cavernous sinus malignant lymphoma treated by gamma knife radiosurgery: case report and review of the literature. Surg Neurol. 1996;46:272-8; discussion 8-9.

5. Roman-Goldstein SM, Jones A, Delashaw JB, et al. Atypical central nervous system lymphoma at the cranial base: report of four cases. Neurosurgery. 1998;43:613-5; discussion 5-6. 